\title{
LOCALIZATION OF THE ZEROS OF THE PERMANENT OF A CHARACTERISTIC MATRIX ${ }^{1}$
}

\author{
P. M. GIBSON
}

AbSTRACT. It is shown that many bounds for the eigenvalues of a complex matrix $A$ are also bounds for the zeros of the permanent of the characteristic matrix of $A$.

If $A=\left(a_{i j}\right)$ is an $n$-square nonnegative matrix, let $\Phi(A)$ be the set of all $n$-square complex matrices $B=\left(b_{i j}\right)$ for which $\left|b_{i j}\right|=a_{i j}$ whenever $i \neq j$, and let $\Psi(A)$ be the subset of $\Phi(A)$ consisting of all $B$ for which $\left|b_{i i}\right|=a_{i i}$ for all $i$. This note is based on the following.

THEOREM 1. If $A$ is an $n$-square nonnegative matrix then

$$
\operatorname{det} B \neq 0 \quad \forall B \in \Psi(A) \Rightarrow \text { per } B \neq 0 \quad \forall B \in \Psi(A) \text {. }
$$

Proof. As Camion and Hoffman [4] have shown, if $\operatorname{det} B \neq 0$ for every $B \in \Psi(A)$, then there exist a permutation matrix $P$ and a nonsingular diagonal matrix $D$ such that $P A D$ is a dominant diagonal matrix. Brenner [1] has shown that the permanent of a dominant diagonal matrix is nonzero. Hence, since $P B D$ is dominant diagonal,

$$
\text { per } B=(\text { per } P B D)(\text { per } D)^{-1} \neq 0 \quad \forall B \in \Psi(A) .
$$

Consideration of the matrix

$$
A=\left[\begin{array}{lll}
2 & 1 & 1 \\
1 & 2 & 1 \\
1 & 1 & 2
\end{array}\right]
$$

shows that the converse of (1) is false.

If $A$ is an $n$-square matrix then each zero of per $(z I-A)$ is called a $p$-root of $A$. Denote the set of all eigenvalues and the set of all p-roots of $A$ by $\Lambda(A)$ and $\Pi(A)$, respectively. Let $\delta(A)$ be the diagonal of the matrix $A$. An admissible mapping $\sigma$ of a set $S$ of $n$-square complex matrices is a

Received by the editors January 5, 1971 .

AMS 1970 subject classifications. Primary 15A15, 15A42; Secondary 15A48.

Key words and phrases. Permanents, determinants, dominant diagonal matrices, bounds for eigenvalues, spectral radius, nonnegative matrices.

${ }^{1}$ This research was supported in part by the National Science Foundation and the Research Committee of the University of Alabama in Huntsville.

(c) American Mathematical Society 1972 
mapping of $S$ into the power set of the complex numbers such that if $A, B \in S$ with $\delta(A)=\delta(B)$ then $\sigma(A)=\sigma(B)$. We have the following.

THEOREM 2. If $A$ is an $n$-square nonnegative matrix and $\sigma$ is an admissible mapping of $\Phi(A)$, then

$$
\Lambda(B) \subset \sigma(B) \quad \forall B \in \Phi(A) \Rightarrow \Pi(B) \subset \sigma(B) \quad \forall B \in \Phi(A) .
$$

Proof. Let $B=\left(b_{i j}\right) \in \Phi(A), z \notin \sigma(B)$, and let $A^{\prime}$ be the $n$-square nonnegative matrix obtained from $A=\left(a_{i j}\right)$ by replacing each $a_{i i}$ by $\left|z-b_{i i}\right|$. Let $C \in \Psi^{(}\left(A^{\prime}\right)$. Then, since $z I-B \in \Psi^{\circ}\left(A^{\prime}\right)$, there exists a unitary diagonal matrix $D$ such that $\delta(D C)=\delta(z I-B)$. Hence, there exists $B^{\prime} \in \Phi(A)$ such that $\delta\left(B^{\prime}\right)=\delta(B)$ and $D C=z I-B^{\prime}$. Since $z \notin \sigma(B)=$ $\sigma\left(B^{\prime}\right)$,

$$
\operatorname{det} C=(\operatorname{det} D)^{-1} \operatorname{det} D C=(\operatorname{det} D)^{-1} \operatorname{det}\left(z I-B^{\prime}\right) \neq 0 .
$$

Hence, det $C \neq 0$ for every $C \in \Psi\left(A^{\prime}\right)$. Therefore, since $z I-B \in \Psi\left(A^{\prime}\right)$, by Theorem 1 , per $(z I-B) \neq 0$.

Many estimates for the eigenvalues of a matrix have been discovered (see for example, [2], [5], and their references). It follows from Theorem 2 that many of these are also estimates for the $p$-roots of the matrix. For example, we have the following.

Corollary 1. If $A=\left(a_{i j}\right)$ is an n-square complex matrix, $0 \leqq p \leqq 1$, and

$$
R_{i}=\sum_{k \neq i}\left|a_{i k}\right|, \quad C_{i}=\sum_{k \neq i}\left|a_{k i}\right|, \quad i=1, \cdots, n,
$$

then each p-root of $A$ lies in at least one of the ovals

$$
\left\{z:\left|z-a_{i i}\right|\left|z-a_{j j}\right| \leqq\left(R_{i} R_{j}\right)^{p}\left(C_{i} C_{j}\right)^{1-p}\right\}, \quad 1 \leqq i<j \leqq n .
$$

Proof. Let $B=\left(b_{i j}\right)$ be an $n$-square nonnegative matrix with $b_{i j}=$ $\left|a_{i j}\right|$ whenever $i \neq j$. For each $D=\left(d_{i j}\right) \in \Phi(B)$, let

$$
\sigma(D)=\bigcup_{1 \leqq i<j \leqq n}\left\{z:\left|z-d_{i i}\right|\left|z-d_{j j}\right| \leqq\left(R_{i} R_{j}\right)^{p}\left(C_{i} C_{j}\right)^{1-p}\right\} .
$$

Using a theorem on eigenvalues due to Ostrowski [6], we have

$$
\Lambda(D) \subset \sigma(D) \quad \forall D \in \Phi(B) .
$$

Hence, since $A \in \Phi(B)$, from Theorem 2, $\Pi(A) \subset \sigma(A)$.

THEOREM 3. Let $A$ be an n-square nonnegative matrix, and let $S$ be a set of complex numbers. Then

$$
\Lambda(B) \subset S \quad \forall B \in \Psi(A) \Rightarrow \Pi(B) \subset S \quad \forall B \in \Psi(A) .
$$


Proof. Let $B \in \Psi(A)$. For each $C \in \Phi(A)$, let $\sigma(C)$ be $S$ or the set of all complex numbers according to whether $\delta(C)=\delta(B)$ or $\delta(C) \neq \delta(B)$. Now apply Theorem 2 to get $\Pi(B) \subset \sigma(B)=S$.

Let $\rho(A)$ be the spectral radius of the matrix $A$. Theorem 3 and a wellknown bound for the eigenvalues of a matrix give the following.

Corollary 2. Let $A=\left(a_{i j}\right)$ and $B=\left(b_{i j}\right)$ be $n$-square matrices. If $\pi$ is a p-root of $A$ and $\left|a_{i j}\right| \leqq b_{i j}$ for $i, j=1, \cdots, n$, then $|\pi| \leqq \rho(B)$.

From this corollary we obtain the following result of Brenner and Brualdi [3].

COROLlaRY 3. If $A$ is an n-square nonnegative matrix and $\pi$ is a p-root of $A$, then $|\pi| \leqq \rho(A)$.

\section{REFERENCES}

1. J. L. Brenner, Relations among the minors of a matrix with dominant principal diagonal, Duke Math. J. 26 (1959), 563-567. MR 22 \#1590.

2. - Geršgorin theorems, regularity theorems, and bounds for determinants of partitioned matrices, SIAM J. Appl. Math. 19 (1970), 443-450.

3. J. L. Brenner and R. A. Brualdi, Eigenschaften der Permanentefunktion, Arch. Math. (Basel) 18 (1967), 585-586. MR 36 \#3795.

4. P. Camion and A. J. Hoffman, On the nonsingularity of complex matrices, Pacific J. Math. 17 (1966), 211-214. MR 33 \#129.

5. M. Marcus and $\mathrm{H}$. Minc, $A$ survey of matrix theory and matrix inequalities, Allyn and Bacon, Boston, Mass., 1964. MR 29 \#112.

6. A. Ostrowski, Über das Nichtverschwinden einer Klasse von Determinanten und die Lokalisierung der charakteristischen Wurzeln von Matrizen, Compositio Math. 9 (1951), 209-226. MR 13, 524.

Department of Mathematics, University of Alabama, Huntsville, Alabama 35807 